\title{
EUCLIDEAN $n$-PLANES IN PSEUDO-EUCLIDEAN SPACES AND DIFFERENTIAL GEOMETRY OF CARTAN DOMAINS
}

\author{
BY YUNG-CHOW WONG \\ Communicated by W. T. Martin, September 12, 1968
}

1. Introduction. The Cartan domains, which we shall define in 83, include among them the four general types of (irreducible) bounded symmetric domains, first studied by E. Cartan [2], [3]. An (essentially unique) invariant Riemannian metric-the Bergman metric-exists on each of these bounded symmetric domains, and the resulting differential geometry has been studied by Siegel [7], Hua [4], [5], Look [6] and others.

In this note we describe how the differential geometry of Cartan domains can be studied neatly and effectively through a study of the Euclidean $n$-planes in a pseudo-Euclidean $(n+m)$-space of index $m$. Our results include a geometric interpretation of the Bergman metric, the theorem that domains of the second and third types are totally geodesic submanifolds of a domain of the first type, and ranges of value of the sectional curvature. Only a brief description of the method and results will be given here. The reader will find in this and three other notes [8], [9], [10] the essence of the differential geometry of the eight nonspecial types of irreducible Hermitian symmetric spaces (see [1]).

2. Euclidean $n$-planes in a pseudo-Euclidean space. Let $F$ be the field $R$ of real numbers, the field $C$ of complex numbers, or the field $H$ of real quaternions. Let $\{1, i, j, k\}$ be the usual basis of $F$ over $R$. If $\xi=a_{0}+a_{1} i+a_{2} j+a_{3} k$, then

$$
\xi=a_{0}-a_{1} i-a_{2} j-a_{3} k, \quad \xi=a_{0}+a_{1} i+a_{2} j-a_{3} k
$$

are two conjugates of $\xi$. If $A$ is an $n \times m$ matrix with elements in $F$, we denote by $A^{*}, A^{\tau}$ the two respective conjugate transposes of $A$. For a square matrix $A$, if $A^{*}=A, A^{\tau}=A$, or $A^{r}=-A$, we say, respectively, that $A$ is Hermitian, $\tau$-symmetric, or $\tau$-skew-symmetric. Clearly, for $F=R$ or $C, \tau$-symmetry and $\tau$-skew-symmetry are the ordinary symmetry and ordinary skew-symmetry.

By definition, a pseudo-Euclidean space $F_{(m)}^{n+m}$ (of index $m$ ) is an $(n+m)$-dimensional left vector space over $F$ provided with a (Hermitian) inner product $\langle$,$\rangle such that there exist n$-planes (i.e. $n$-dimensional vector subspaces), but not $(n+1)$-planes, on which the induced 
inner product is positive definite. In $F_{(m)}^{n+m}$, natural systems of rectangular coordinates exist such that if

$$
(x, y) \equiv\left(x_{1}, \cdots, x_{n} ; x_{n+1}, \cdots, x_{n+m}\right)
$$

are the coordinates of a vector $u$, then $\langle u, u\rangle=x x^{*}-y y^{*}$.

An important case of $F_{(m)}^{n+m}$ is the real hyperbolic plane $R_{(1)}^{2}$. If $u, v$ are two vectors of $R_{(1)}^{2}$ such that $\langle u, u\rangle,\langle u, v\rangle$ and $v,\langle v\rangle$ are all $>0$, then there exists a unique real number $\theta$, called the angle between $u$ and $v$, defined by

$$
\cosh \theta=\langle u, v\rangle /(\langle u, u\rangle\langle v, v\rangle)^{1 / 2}, \quad 0 \leqq \theta<+\infty .
$$

In $F_{(m)}^{n+m}$, an $n$-plane is called a Euchdean $n$-plane if the inner product induced on it is positive definite. Let $A$ an $\mathrm{d} B$ be two Euclidean $n$-planes in $F_{(m)}^{n+m}$. We can prove that, if $\boldsymbol{u}$ is a nonzero vector in $\boldsymbol{A}$, and $v$ the orthogonal projection of $u$ in $B$, then

(i) $v \neq 0$; and

(ii) either $v=u$, or $u$ and $v$ span an $R_{(1)}^{2}$ and $\langle u, v\rangle>0$. Thus there exists a unique angle between any nonzero vector $u$ in $A$ and its projection in $B$, and we can define the angles between $A$ and $B$ as the stationary values of the angle between $u$ and its projection in $B$ as $u$ runs through $\boldsymbol{A}$. With this done, the development of the geometry of Euclidean $n$-planes in the pseudo-Euclidean space $F_{(m)}^{n+m}$ proceeds parallelly to that of the geometry of $n$-planes in the Euclidean space $F^{n+m}$. The definitions and results in $[8, \S 2]$ can be carried over without difficulty. For example, we can prove that there are $n$ angles between two Euclidean $n$-planes $A$ and $B$ in $F_{(m)}^{n+m}$ and they completely determine the relative position of $\boldsymbol{A}$ and $B$; moreover, there are orthogonal frames of angle-planes (i.e., real hyperbolic planes containing the angles) associated with $A$ and $B$, and so on.

3. The Cartan domains. The first Cartan domain, denoted by $D_{\mathrm{I}}\left(F_{(m)}^{n+m}\right)$, is the manifold of Euclidean $n$-planes in $F_{(m)}^{n+m}$. Let $(x, y)$ be a natural system of rectangular coordinates in $F_{(m)}^{n+m}$. We can prove that an $n$-plane in $F_{(m)}^{n+m}$ is a Euclidean $n$-plane iff it has an equation of the form $y=x Z$, where $Z$ is an $n \times m$ matrix such that $I-Z Z^{*}>0$ (i.e., the Hermitian matrix $I-Z Z^{*}$ is positive definite). Thus, $D_{\mathrm{I}}\left(F_{(m)}^{n+m}\right)$ can be identified with the space of all $n \times m$ matrices $Z$ such that $I-Z Z^{*}>0$. The elements of $Z$ serve as coordinates in $D_{\mathrm{I}}\left(F_{(m)}^{n+m}\right)$.

In $F_{(n)}^{2 n}$, the equation

$$
x \tilde{y}^{r}-y \bar{x}^{r}=0,
$$

where $(x, y)$ and $(\tilde{x}, \tilde{y})$ are the coordinates of two vectors in $F_{(n)}^{2 n}$, determines a null system; and the equation 


$$
x y^{\tau}+y x^{\tau}=0
$$

determines a hyperquadric. The second Cartan domain, denoted by $D_{\mathrm{II}}\left(F_{(n)}^{2 n}\right)$, is the manifold of all the Euclidean $n$-planes in $F_{(n)}^{2 n}$ each of which is self-polar with respect to the null system (3.1). The third Cartan domain, denoted by $D_{\mathrm{III}}\left(F_{(n)}^{2 n}\right)$, is the manifold of all the Euclidean $n$-planes in $F_{(n)}^{2 n}$ each lying entirely in the hyperquadric (3.2). It is easy to see that $D_{\mathrm{II}}\left(F_{(n)}^{2 n}\right)$ (resp. $\left.D_{\mathrm{III}}\left(F_{(n)}^{2 n}\right)\right)$ can be identified with the space of all $n \times n \tau$-symmetric (resp. $\tau$-skew-symmetric) matrices $Z$ such that $I-Z Z^{*}>0$.

The group of motions in $F_{(m)}^{n+m}$ induces on $D_{\mathrm{I}}\left(F_{(m)}^{n+m}\right)$ a transitive group $U_{\mathrm{I}}\left(F_{(m)}^{n+m}\right)$ of motions. The subgroups of $U_{\mathrm{I}}\left(F_{(n)}^{2 n}\right)$ which leave $D_{\mathrm{II}}\left(F_{(n)}^{2 n}\right)$ and $D_{\mathrm{III}}\left(F_{(n)}^{2 n}\right)$ respectively invariant are also transitive. Thus Cartan domains are homogeneous spaces; in fact, they are symmetric spaces.

We observe that the Cartan domains $D_{\mathrm{I}}\left(C_{(m)}^{n+m}\right), D_{\mathrm{II}}\left(C_{(n)}^{2 n}\right), D_{\mathrm{III}}\left(C_{(n)}^{2 n}\right)$ and $D_{\mathrm{I}}\left(R_{(n)}^{2+n}\right)$ are precisely the four general types of irreducible bounded symmetric domains (see [3], [5, p. 5] and [1, p. 489]).

\section{Invariant Riemannian metric and geodesics in Cartan domains.}

THEOREM 4.1. The sum of squares of the $n$ angles between two consecutive Euclidean n-planes in $F_{(m)}^{n+m}$ provides $D_{\mathrm{I}}\left(F_{(m)}^{n+m}\right)$ with an invariant Riemannian metric whose analytic expression is

$$
d s^{2}=\operatorname{Re} \operatorname{Tr}\left[\left(I-Z Z^{*}\right)^{-1} d Z\left(I-Z^{*} Z\right)^{-1} d Z^{*}\right],
$$

where $\operatorname{Re} \operatorname{Tr}$ denotes the real part of the trace. In particular, for $F=C$, this reduces to the Bergman metric

$$
d s^{2}=\operatorname{Tr}\left[\left(I-Z Z^{*}\right)^{-1} d Z\left(I-Z^{*} Z\right)^{-1} d Z^{*}\right] .
$$

We have thus a nice geometric interpretation of the Bergman metric on bounded symmetric domains of the first type.

TheOREM 4.2. The differential equation of the geodesics in $D_{\mathrm{I}}\left(F_{(m)}^{n+m}\right)$ is

$$
Z+2 Z Z^{*}\left(I-Z Z^{*}\right)^{-1} Z=0,
$$

where the dots denote derivatives with respect to the arc length $s$.

Theorem 4.3. Any geodesic in $D_{\mathrm{I}}\left(F_{(m)}^{n+m}\right), D_{\mathrm{II}}\left(F_{(n)}^{2 n}\right)$, or $D_{\mathrm{III}}\left(F_{(n)}^{2 n}\right)$ is congruent respectively to

(i) $Z=\left[\begin{array}{cc}Z_{1}(s) & 0 \\ 0 & 0\end{array}\right], \quad Z_{1}(s)=\operatorname{diag}\left(\tanh \tau_{1} s, \cdots, \tanh \tau_{r} s\right)$,

(ii) $Z=Z(s)=\operatorname{diag}\left( \pm \tanh \tau_{1} s, \cdots, \pm \tanh \tau_{r} s, 0, \cdots, 0\right)$, 
or

$$
\text { (iii) } \begin{aligned}
Z=Z(s)=\operatorname{diag}\left\{\tanh \tau_{1} s\left[\begin{array}{cc}
\cos \omega_{1} k & \sin \omega_{1} \\
-\sin \omega_{1} & -\cos \omega_{1} k
\end{array}\right], \cdots,\right. \\
\tanh \tau_{q} s\left[\begin{array}{cc}
\cos \omega_{q} k & \sin \omega_{q} \\
-\sin \omega_{q} & -\cos \omega_{q} k
\end{array}\right], \\
\left. \pm\left(\tanh \tau_{2 q+1} s\right) k, \cdots, \pm\left(\tanh \tau_{r} s\right) k, 0, \cdots, 0\right\}
\end{aligned}
$$

where in (i) and (ii) the $\tau^{\prime}$ 's are positive numbers such that $\left(\tau_{1}\right)^{2}+\ldots$ $+\left(\tau_{r}\right)^{2}=1$, and in (iii) the $\tau$ 's and $\omega$ 's are positive numbers such that $2\left(\tau_{1}\right)^{2}+\cdots+2\left(\tau_{q}\right)^{2}+\left(\tau_{2 q+1}\right)^{2}+\cdots+\left(\tau_{r}\right)^{2}=1$ and each of the $\omega$ 's is $<\pi$.

TheOREM 4.4. $A C^{2}$-curve $\Gamma$ in $D_{\mathrm{I}}\left(F_{(m)}^{n+m}\right)$ is a geodesic iff when it is viewed as 1-parameter family of Euclidean $n$-planes in $F_{(m)}^{n+m}$,

(a) all the pairs of Euclidean $n$-planes of $\Gamma$ have common angleplanes, and

(b) the $n$ angles (arranged in a definite order) between any two Euclidean $n$-planes of $\Gamma$ are proportional to a fixed set of (nonnegative) constants.

THEOREM 4.5. (a) There is a unique geodesic segment joining any two points in $D_{\mathrm{I}}\left(F_{(m)}^{n+m}\right)$ (for $F=C$, this is known; see [6]).

(b) The geodesic segment joining the two points $A$ and $B$ in $D_{\mathrm{I}}\left(F_{(m)}^{n+m}\right)$ is of length $\left[\Sigma\left(\theta_{i}\right)^{2}\right]^{1 / 2}$, where $\theta_{i}$ are the $n$ angles between the Euclidean $n$-planes $A$ and $B$ in $F_{(m)}^{n+m}$.

The geodesics in $D_{\mathrm{II}}\left(F_{(n)}^{2 n}\right)$ and $D_{\mathrm{III}}\left(F_{(n)}^{2 n}\right)$ also have the properties stated in Theorems 4.4 and 4.5. However, the following inclusive theorem can be proved.

Theorem 4.6. $D_{\mathrm{II}}\left(F_{(n)}^{2 n}\right)$ and $D_{\mathrm{III}}\left(F_{(n)}^{2 n}\right)$ are totally geodesic submanifolds of $D_{\mathrm{I}}\left(F_{(n)}^{2 n}\right)$.

Two Euclidean $n$-planes in $F_{(n)}^{2 n}$ are said to be mutually isoclinic if the angles between them are all equal. We can prove

TheOREM 4.7. Any maximal set of mutually isoclinic Euclidean $n$-planes in $F_{(n)}^{2 n}$ when viewed as a subset of $D_{\mathrm{I}}\left(F_{(n)}^{2 n}\right)$ is a totally geodesic submanifold which is analytically isometric with the pseudo-sphere of curvature $-4 / n$.

5. Sectional curvatures of the Cartan domains. Explicit expression for the sectional curvature of $D_{\mathrm{I}}\left(F_{(m)}^{n+m}\right)$ differs from that of the Grass- 
mann manifold $G_{n}\left(F^{n+m}\right)$ as given in $[10, \S 3]$ by only a sign. From this expression, we can obtain the ranges of value of the sectional curvature of all the Cartan domains, listed in the following table.

SECTIONAL CURVATURE $K$

\begin{tabular}{|c|c|c|}
\hline Cartan Domain & & Range of Value of $K$ \\
\hline$D_{\mathrm{I}}\left(R_{(m)}^{n+m}\right)$ & $\left.\begin{array}{rl}n & =1, m=1 \\
n & =1, m \geqq 2 \\
\text { or } n & \geqq 2, m=1 \\
n & \geqq 2, m \geqq 2\end{array}\right\}$ & $\begin{array}{l}\text { Sectional curvature not defined } \\
\qquad \begin{array}{c}K=-1 \\
-2 \leqq K \leqq 0\end{array}\end{array}$ \\
\hline$D_{\mathrm{I}}\left(C_{(m)}^{n+m}\right), D_{\mathrm{I}}\left(H_{(m)}^{n+m}\right)$ & $\left.\begin{array}{rl}n & =1, m=1 \\
n & =1, m \geqq 2 \\
\text { or } n & \geqq 2, m=1 \\
n & \geqq 2, m \geqq 2\end{array}\right\}$ & $\begin{aligned} K & =-4 \\
-4 & \leqq K \leqq-1 \\
-4 & \leqq K \leqq 0\end{aligned}$ \\
\hline$D_{\mathrm{II}}\left(R_{(n)}^{2 n}\right)$ & $n \geqq 2$ & $-2 \leqq K \leqq 0$ \\
\hline$D_{\mathrm{II}}\left(C_{(n)}^{2 n}\right), D_{\mathrm{II}}\left(H_{(n)}^{2 n}\right)$ & $n \geqq 2$ & $-4 \leqq K \leqq 0$ \\
\hline$D_{\mathrm{III}}\left(R_{(n)}^{2 n}\right)$ & $\begin{array}{l}n=2 \\
n=3 \\
n \geqq 4\end{array}$ & $\begin{array}{l}\text { Sectional curvature not defined } \\
\qquad \begin{array}{c}K=-\frac{1}{2} \\
-1 \leqq K \leqq 0\end{array}\end{array}$ \\
\hline$D_{\mathrm{III}}\left(C_{(n)}^{2 n}\right)$ & $\begin{array}{l}n=2 \\
n=3 \\
n \geqq 4\end{array}$ & $\begin{aligned} & K=-2 \\
- & 2 \leqq K \leqq-\frac{1}{2} \\
- & 2 \leqq K \leqq 0\end{aligned}$ \\
\hline$D_{\mathrm{III}}\left(H_{(n)}^{2 n}\right)$ & $n \geqq 2$ & $-2 \leqq K \leqq 0$ \\
\hline
\end{tabular}

ADDED IN PROOF. The following results can be proved:

\section{The Cartan domains}

$$
\begin{array}{lll}
D_{\mathrm{I}}\left(R_{(m)}^{n+m}\right), & D_{\mathrm{I}}\left(C_{(m)}^{n+m}\right), & D_{\mathrm{I}}\left(H_{(m)}^{n+m}\right) ; \\
D_{\mathrm{II}}\left(R_{(n)}^{2 n}\right), & D_{\mathrm{II}}\left(C_{(n)}^{2 n}\right), & D_{\mathrm{II}}\left(H_{(n)}^{2 n}\right) ; \\
D_{\mathrm{III}}\left(R_{(n)}^{2 n}\right), & D_{\mathrm{III}}\left(C_{(n)}^{2 n}\right), & D_{\mathrm{III}}\left(H_{(n)}^{2 n}\right)
\end{array}
$$

have respectively the scalar curvatures

$$
\begin{array}{lll}
-n m(n+m-2), & -4 n m(n+m), & -16 n m(n+m+1) ; \\
-\frac{1}{2} n(n-1)(n+2), & -2 n(n+1)^{2}, & -4 n(n+1)(2 n+1) ; \\
-\frac{1}{2} n(n-2)(n-1), & -2 n(n-1)^{2}, & -4 n(n-1)(2 n+1) .
\end{array}
$$


Moreover, with the exception of $D_{\mathrm{II}}\left(R_{(n)}^{2 n}\right)$ and $D_{\mathrm{III}}\left(H_{(n)}^{2 n}\right)$, they are all Einslein spaces.

The author is deeply indebted to Professor S. S. Chern for the many helpful discussions he had with him at Berkeley in the Spring term of 1967-discussions that eventually led to the results outlined in this note. The author is also grateful to Professor Chern's colleagues, especially Professors S. Kobayashi, J. A. Wolf and F. W. Warner, for several stimulating conversations concerning differential geometry of Grassmann manifolds.

\section{REFERENCES}

1. E. Calabi and E. Vesentini, On compact locally symmetric Kähler manifolds, Ann. of Math. (2) 71 (1960), 472-507.

2. E. Cartan, Sur certaines formes riemanniennes remarquables des geometries d groupe fondamental simple, Ann. Sci. École Norm. Sup. 44 (1927), 345-467.

3. - Sur les domaines bornès homogènes de l'espace de $n$ variables complexes, Abh. Math. Sem. Univ. Hamburg 11 (1935), 116-162.

4. L. K. Hua, On the theory of automorphic functions of a matrix variable. I: Geometrical basis, Amer. J. Math. 66 (1944), 470-488.

5. - Harmonic analysis of functions of several complex variables in the classical domains, Transl. Math. Monographs, vol. 6, Amer. Math. Soc., Providence, R.I., 1963.

6. K. H. Look, Classical manifolds and classical domains, Shanghai, 1963 (in Chinese).

7. C. L. Siegel, Symplectic geometry, Amer. J. Math. 55 (1943), 1-86.

8. Y. C. Wong, Differential geometry of Grassmann manifolds, Proc. Nat. Acad. Sci. U.S.A. 57 (1967), 589-594.

9. - Conjugate loci in Grassmann manifolds, Bull. Amer. Math. Soc. 74 (1968), 240-245.

10. - Sectional curvature of Grassmann manifolds, Proc. Nat. Acad. Sci. U.S.A. 58 (1968), 75-79.

University OF Hong Kong, Hong Kong 